Pacific Journal of Mathematics

ON TOPOLOGICAL ANALOGUES OF LEFT THICK SUBSETS 


\title{
ON TOPOLOGICAL ANALOGUES OF LEFT THICK SUBSETS IN SEMIGROUPS
}

\author{
JAMES C. S. WONG
}

\begin{abstract}
We discuss the relation among various topological analogues of left thickness in semigroups and their connection with left invariant means for locally compact separately continuous semigroups. Until now, most results in this direction have been obtained for only jointly continuous semigroups. However, an important convolution formula found recently by this author made the transition to separately continuous cases possible.
\end{abstract}

1. Introduction. Let $S$ be a semigroup and $T$ a subset of $S$. $T$ is called left thick if for each finite set $F \subset S$, there is some $s \in S$ such that $F s \subset T$. In 1965, T. Mitchell obtained the following interesting results:

THEOREM 1.1 (Mitchell [7]). Let $S$ be a left amenable semigroup and $T$ a subset of $S$, then $T$ is left thick iff there is a left invariant mean $m$ on $S$ such that $m\left(\xi_{T}\right)=1$ where $\xi_{T}$ is the characteristic function of $T$.

THEOREM 1.2 (Mitchell [7]). If $T$ is a left thick subsemigroup of a semigroup $S$, then $S$ is left amenable iff $T$ is left amenable.

Since then, various attempts have been made to obtain topological analogues and extensions of these concepts and results to locally compact semigroups (with jointly continuous multiplication) with only partial success (see Day [3], [4] and Wong [10], [11]). In fact, in these attempts, a topological analogue of one or the other (but not both) of Mitchell's theorems was found. The purpose of this paper is two-fold. First, we introduce a "suitable" topological analogue of left thickness and extend both of Mitchell's results. Second, we shall do it in the more general setting of locally compact separately continuous semigroups because of an important convolution formula obtained recently by this author for such semigroups (see Wong [12] and $\S 2$ below).

$\S 2$. Notations and background. For notations and definitions in analysis on locally compact (Hausdorff) semigroups, we shall follow [11] (to which the present paper is a sequel) except that we are now dealing with a locally compact separately continuous semigroup $S$. Although all the results cited in the references here are 
for jointly continuous semigroups (or compact separately continuous ones), many of them (in particular, those we are going to need here) can be carried over to general separately continuous semigroups. We shall discuss this briefly here and where appropriate, special remarks with respect to this will be made below.

As usual, let $M(S)$ be the measure algebra with convolution product and $M_{0}(S)$ the probability measures. Recently, this author has obtained the following convolution formula:

$$
\int f d \mu * \nu=\iint f(x y) d \mu(x) d \nu(y)=\iint f(x y) d \nu(y) d \mu(x)
$$

for all $f \in L_{1}(|\mu| *|\nu|), \mu, \nu \in M(S)$. (See Wong [12].) It follows that $M_{0}(S)$ is a convolution semigroup (algebraically) and that

$$
\operatorname{support}(\mu * \nu)=[\operatorname{support} \mu \cdot \operatorname{support} \nu]^{-}
$$

if $\mu, \nu \in M_{0}(S)$.

As a result, if $S$ is jointly continuous, $\mu * \nu$ has compact support whenever $\mu, \nu$ do. In general, this may not be the case except for example when $\mu=\delta_{a}$ is the Dirac measure and $\nu$ has compact support. Then $\delta_{a} * \nu$ has compact support (=a. support $\nu$ ).

Also, the functions $x \rightarrow f(x y), y$ fixed and $x \rightarrow \int f(x y) d \nu(y)$, though continuous, need not be in $C_{0}(S)$ if $f$ is in $C_{0}(S)$, the continuous functions on $S$ which vanish at infinity. Thus $M_{0}(S)$ need not be a topological semigroup under the weak* topology of $M(S)=C_{0}(S)^{*}$. Despite this apparent setback for separately continuous semigroups, it should however be remarked that for example the construction used in Wong [11, Lemma 3.1, p. 296] is valid for separately continuous semigroups since it requires only that $M_{0}(S)$ be a semigroup.

Now let $T$ be a Borel subset of $S$. Consider the following conditions on $T$ :

$(T L S)$ For "each $K \subset S$ compact, there is some $\mu \in M_{0}(S)$ such that $\nu * \mu(T)=1$ for any $\nu \in M_{0}(S)$ with $\nu(K)=1$. [Can assume $\mu(T)=1$.]

$(T L T)$ For each $\varepsilon>0, K \subset S$ compact, there is some $\mu \in M_{0}(S)$ such that $\nu * \mu(T)>1-\varepsilon$ for any $\nu \in M_{0}(S)$ with $\nu(K)=1$. [Can assume that $\mu$ has compact support and $\mu(T)>1-\varepsilon$.]

$\left(T L L_{1}\right)$ For each $\varepsilon>0$ and $\nu \in M_{0}(S)$ with compact support, there is some $\mu$ in $M_{0}(S)$ with compact support such that $\nu * \mu(T)>$ $1-\varepsilon$.

$(T L L)$ For each $\varepsilon<0$ and $\nu \in M_{0}(S)$ with compact support, there is some $s \in S$ such that $\nu * \delta_{s}(T)>1-\varepsilon$ where $\delta_{s}$ is the Dirac measure at $s$.

$(L T)$ For each $F \subset S$ finite, there is some $s \in S$ such that $F s \subset$ 
$T$ [Can assume $s \in T$.] (This is Mitchell's definition of left thickness.) and

(*) For each $\varepsilon>0$ and $\nu \in M_{0}(S)$ with compact support, there is some $\mu \in M_{0}(S)$ with compact support such that $\mu(T)>1-\varepsilon$ and $\nu * \mu(T)>1-\varepsilon$.

$T$ is called topological left substantial if $T$ satisfies (TLS). In Wong [10], it is proved that if $T$ is a (locally compact Borel) topological left substantial subsemigroup of $S$, then $T$ is topological left amenable iff $S$ is. This is a topological analogue and extension of Theorem 1.2. Also condition (TLS) remains unchanged if we require the measure $\mu$ to satisfy the additional assumption that $\mu(T)=1$. The proof can be found in [10]. Since similar situations will frequently occur again below, we present the proof here for completeness. As in [10], if $\phi \neq K \subset S$ compact is given, choose $k \in K$ and let $K_{1}=K k \cup\{k\}$ which is also compact. There is some $\mu_{1} \in M_{0}(S)$ such that $\nu_{1} * \mu_{1}(T)=1$ if $\nu_{1} \in M_{0}(S)$ and $\nu_{1}\left(K_{1}\right)=1$. Consider $\mu=\delta_{k} * \mu_{1} \in M_{0}(S) . \quad \mu(T)=1$ since $\delta_{k}\left(K_{1}\right)=1$. Moreover, if $\nu \in M_{0}(S)$ and $\nu(K)=1$, then

$$
\begin{aligned}
\nu * \mu(T)=\left(\nu * \delta_{k}\right) & * \mu_{1}(T)=1 \text { since } \nu * \delta_{k}\left(K_{1}\right) \\
= & \int_{K} \xi_{K_{1}}(x k) d \nu(x)=\nu(K)=1 .
\end{aligned}
$$

On the other hand, $T$ is called topological left thick if $T$ satisfies $(T L T)$. It is proved in Wong [11] that if $S$ is uniform strong topological left amenable (hence topological left amenable), then $T$ is topological left thick iff there is a topological left invariant mean $M$ on $M(S)^{*}$ such that $M\left(\chi_{T}\right)=1$ where $\chi_{T}$ is the characteristic functional of $T$ in $S$ (see [11] for more details). This is a topological analogue and extension of Theorem 1.2. Condition (TLT) remains unchanged if we require the measure $\mu$ to satisfy the additional assumptions that $\mu$ has compact support and $\mu(T)>1-\varepsilon$. For if $\varepsilon>o$ and $K \subset S$ compact are given, there is some $\mu \in M_{0}(S)$ such that $\nu * \mu(T)>1-\varepsilon / 2$ for all $\nu \in M_{0}(S)$ with $\nu(K)=1$. Since the measures in $M_{0}(S)$ with compact supports are norm dense in $M_{0}(S)$, we can choose $\mu_{1} \in M_{0}(S)$ with compact support such that $\left\|\mu-\mu_{1}\right\|<\varepsilon / 2$, then

$$
\left|\left(\nu * \mu_{1}-\nu * \mu\right)(T)\right| \leqq\left\|\nu * \mu_{1}-\nu * \mu\right\|<\frac{\varepsilon}{2}
$$

and $\nu * \mu_{1}(T)>1-\varepsilon$ for all $\nu \in M_{0}(S)$ with $\nu(K)=1$. Next, suppose the pair $(\varepsilon, K)$ is given and $K \neq \phi$. Choose $k \in K$ and let $K_{1}=K k \cup$ $\{k\}$ which is compact. By the above argument, there is some $\mu_{2} \in$ 
$M_{0}(S)$ with compact support such that

$$
\tau * \mu_{2}(T)>1-\varepsilon \text { for all } \tau \in M_{0}(S)
$$

with $\tau\left(K_{1}\right)=1$. Consider $\mu_{3}=\delta_{k} * \mu_{2} \in M_{0}(S)$, which has compact support $\left(=k \cdot\right.$ support $\left.\mu_{2}\right), \quad \mu_{3}(T)=\delta_{k} * \mu_{2}(T)>1-\varepsilon$ since $\delta_{k}\left(K_{1}\right)=1$. Moreover, if $\nu \in M_{0}(S)$ and $\nu(K)=1$, then $\nu * \mu_{3}(T)=\left(\nu * \delta_{k}\right) * \mu_{2}(T)>$ $1-\varepsilon$ since $\nu * \delta_{k}\left(K_{1}\right)=\int_{K} \xi_{K_{1}}(x k) d \nu(x)=\nu(K)=1$.

Later, M. Day [4] improves the result in Wong [11, Theorem 4.1, p. 297] by calling $T$ topological left lumpy if $T$ satisfies (TLL) and proves that if $S$ is topological left amenable, then $T$ is topological left lumpy iff there is a topological left invariant mean $M$ on $M(S)^{*}$ such that $M\left(\chi_{T}\right)=1$. Thus for uniform strong topological left amenable semigroups (in particular, any left amenable locally compact group), the concepts of $(T L T)$ and $(T L L)$ are the same.

In general, of course (TLS) implies $(T L T)$ which in turn implies $\left(T L L_{1}\right)$. Also $(T L L)$ and $\left(T L L_{1}\right)$ are equivalent. This is due to Day [4] (under further but redundant assumption). Clearly (TLL) implies $\left(T L L_{1}\right)$. Conversely, if $\nu \in M_{0}(S)$ has compact support and $\nu * \delta_{S}(T) \leqq$ $1-\varepsilon$ for all $s$ in $S$, then $\nu * \mu(T)=\int \nu\left(T s^{-1}\right) d \mu(s)=\int \nu * \delta_{s}(T) d \mu(s) \leqq$ $1-\varepsilon$ for all $\mu \in M_{0}(S)$. Hence $\left(T L L_{1}\right)$ and $(T L L)$ are equivalent.

Also $(T L L)$ implies $(L T)$. The proof is implicit in Day [4]. For given any finite $F \subset S$ with $k$ elements, consider $\nu=1 / k \sum_{\sigma \in F} \delta_{\sigma} \in$ $M_{0}(S)$ with compact support. By $(T L L)$, there is some $s \in S$ such that $\nu * \delta_{s}(T)>1-1 / k$. Hence $\delta_{\sigma s}(T)=1$ for all $\sigma \in F$ or $F s \subset T$.

Finally, condition $\left({ }^{*}\right)$ is somewhere between topological left thickness and topological left lumpiness. Clearly $\left(^{*}\right)$ is formally stronger than $\left(T L L_{1}\right)$. Also $(T L T)$ implies $\left(^{*}\right)$ in view of the above remarks concerning the additional assumptions at the end of the condition $(T L T)$.

This condition $\left(^{*}\right)$ is precisely the "suitable" condition we are looking for in order to extend both Mitchell's results.

\section{Main results.}

THEOREM 3.1. Let $T$ be a Borel subset of a locally compact semigroup $S$ such that $M(S)^{*}$ has a topological left invariant mean. Then the following statements are equivalent:

(1) There is a topological left invariant mean $M$ on $M(S)^{*}$ such that $M\left(\chi_{T}\right)=1$.

(2) $T$ is topological left lumpy (i.e., $T$ satisfies (TLL) or $\left.\left(T L L_{1}\right)\right)$.

(3) T satisfies $\left(^{*}\right)$. 
Proof. Equivalence of (1) and (2) is due to Day [4, Theorem, p. 89]. Since the only difference between conditions $\left(T L L_{1}\right)$ and $\left(^{*}\right)$ is that the measure $\mu$ in $\left(^{*}\right)$ must satisfy the additional assumption that $\mu(T)>1-\varepsilon$, Day's original proof in [4] can easily be adapted to show (1) implies (3). However, we shall present a modification of Day's argument to show that Theorem 3.1 remains valid if the measure $\mu$ in condition $\left({ }^{*}\right)$ is required to satisfy $\mu(T)=1$. Suppose (1) holds and $M$ is a topological left invariant mean such that $M\left(\chi_{T}\right)=1$. Let $\mu_{\alpha}$ be a net in $M_{0}(S)$ with compact supports such that $\mu_{\alpha} \rightarrow M$ weak* in $M(S)^{* *}$. Then $\lim _{\alpha} \mu_{\alpha}(T)=1$ and for each $\nu \in M_{0}(S)$ with compact support,

$$
\nu * \mu_{\alpha}(T)=\chi_{T}\left(\nu * \mu_{\alpha}\right)=\mu_{\alpha}\left(\nu \odot \chi_{T}\right) \longrightarrow M\left(\nu \odot \chi_{T}\right)=M\left(\chi_{T}\right)=1,
$$

since $M$ is topological left invariant.

Define $\tau_{\alpha} \in M^{+}(S)$ by

$$
\int f d \tau_{\alpha}=\int \xi_{T} f d \mu_{\alpha}, f \in C_{0}(S) .
$$

Then $\tau_{\alpha}(B)=\mu_{\alpha}(B \cap T)$ for any Borel set $B$ in $S$. In particular, $\tau_{\alpha}(T)=\mu_{\alpha}(T) \rightarrow 1$. Hence we can assume $\tau_{\alpha}(T) \neq 0$. Let $\nu_{\alpha} \in M_{0}(S)$ be defined by $\nu_{\alpha}=\tau_{\alpha} / \tau_{\alpha}(T)=\tau_{\alpha} / \mu_{\alpha}(T)$. Then for any $f \in C_{0}(S)$, we have

$$
\begin{aligned}
& \left|\int f d \nu_{\alpha}-\int f d \mu_{\alpha}\right|=\left|\frac{1}{\mu_{\alpha}(T)} \int_{T} f d \mu_{\alpha}-\int f d \mu_{\alpha}\right| \\
& \quad \leqq\left|\frac{1}{\mu_{\alpha}(T)} \int_{T} f d \mu_{\alpha}-\int_{T} f d \mu_{\alpha}\right|+\left|\int_{T^{\prime}} f d \mu_{\alpha}\right| \\
& \quad \leqq\|f\|_{u}\left|\frac{1}{\mu_{\alpha}(T)}-1\right|+\|f\|_{u} \mu_{\alpha}\left(T^{\prime}\right) .
\end{aligned}
$$

Hence

$$
\left\|\nu_{\alpha}-\mu_{\alpha}\right\| \leqq\left|\frac{1}{\mu_{\alpha}(T)}-1\right|+\mu_{\alpha}\left(T^{\prime}\right) \longrightarrow 0 \text {. }
$$

Let $\varepsilon>0$ and $\nu \in M_{0}(S)$ with compact support be given, there is some $\alpha_{0}$ (depending on $\varepsilon$ and $\nu$ ) such that

$$
\left\|\nu_{\alpha}-\mu_{\alpha}\right\|<\frac{\varepsilon}{2}
$$

and

$$
\nu * \mu_{\alpha}(T)>1-\varepsilon / 2 \text { if } \alpha \geqq \alpha_{0} .
$$

Hence 


$$
\begin{aligned}
& \left|\nu * \nu_{\alpha}(T)-\nu * \mu_{\alpha}(T)\right| \\
& \quad \leqq\left|\nu * \nu_{\alpha}-\nu * \mu_{\alpha}\right|(T) \\
& \quad \leqq|| \nu_{\alpha}-\mu_{\alpha}||<\frac{\varepsilon}{2} \text { if } \alpha \geqq \alpha_{0} .
\end{aligned}
$$

Consequently

$$
\nu * \nu_{\alpha_{0}}(T)>1-\frac{\varepsilon}{2}-\frac{\varepsilon}{2}=1-\varepsilon .
$$

The measure $\mu=\nu_{\alpha_{0}} \in M_{0}(S)$ has (compact) support $\subset K_{0} \cap T^{-}$where $K_{0}$ is the (compact) support of $\mu_{\alpha_{0}}$ and $\mu$ satisfies the requirements

$$
\mu(T)=1 \text { and } \nu * \mu(T)>1-\varepsilon .
$$

This completes the proof.

REMarks. It should be remarked that Day's result [4, Theorem, p. 89] is for jointly continuous semigroups. However, his proof (and the above adaptation) is actually valid for separately continuous semigroups (see also discussions at the beginning of $\S 2$ ).

Theorem 3.1 is a topological analogue and extension of Theorem 1.1. It is also an improvement of Day's result in [4, Theorem, $p$. 89] (from joint continuity to separate continuity). It also shows that for topological left amenable semigroups conditions (TLL) and $\left({ }^{*}\right)$ are the same. It is not known in general whether condition (*) remains unchanged if we require the measure $\mu$ in $\left(^{*}\right)$ to satisfy $\mu(T)=1$.

To obtain the analogue of Mitchell's second result, we need the following lemmas. From now on, unless otherwise stated explicitly, $T$ is a locally compact Borel subsemigroup of $S$. It is known that if $\mu \in M(S)$, then the restriction $\mu_{T}=\left.\mu\right|_{T}$ of $\mu$ to the Borel subsets of $T$ is a measure in $M(T)$. In fact the correspondence $\mu \rightarrow \mu_{T}$ is an isometric order preserving isomorphism between the subalgebra of all measures $\mu \in M(S)$ with $|\mu|\left(T^{\prime}\right)=0$ and the algebra $M(T)$. Moreover $\mu_{T} \in M_{0}(T)$ if $\mu \in M_{0}(S)$ and $\mu(T)=1$ (see Wong [9] and [4] for details).

LEMmA 3.2. Let $\mu, \nu \in M_{0}(S)$ with $\mu\left(T^{\prime}\right) \leqq \varepsilon$ and $\nu(T)=1$. Then

(1) $|\mu * \nu-\nu|\left(T^{\prime}\right) \leqq \varepsilon$

(2) $|\mu * \nu-\nu|(T) \leqq\left|\mu_{T} * \nu_{T}-\nu_{T}\right|(T)+\varepsilon$.

Consequently

$$
\|\mu * \nu-\nu\| \leqq\left\|\mu_{T} * \nu_{T}-\nu_{T}\right\|+2 \varepsilon .
$$

Proof.

(1) Since $\mu, \nu \geqq 0$ and $\nu\left(T^{\prime}\right)=0$, we have 


$$
\begin{aligned}
\mid \mu * \nu & -\nu\left|\left(T^{\prime}\right) \leqq\right| \mu * \nu\left|\left(T^{\prime}\right)+\right| \nu \mid\left(T^{\prime}\right) \\
& =\mu * \nu\left(T^{\prime}\right)=\iint \xi_{T^{\prime}}(x y) d \mu(x) d \nu(y) \\
& =\int_{T} \int_{T^{\prime}} \xi_{T^{\prime}}(x y) d \mu(x) d \nu(y)+\int_{T} \int_{T^{\prime}} \xi_{T^{\prime}}(x y) d \mu(x) d \nu(y) .
\end{aligned}
$$

Since $T$ is a subsemigroup, $T \cap T^{\prime} y^{-\imath}=\dot{\phi}$ if $y \in T$ and the first integral vanishes. Thus

$$
|\mu * \nu-\nu|\left(T^{\prime}\right) \leqq \nu(T) \cdot \mu\left(T^{\prime}\right) \leqq \varepsilon
$$

(2) If $B \subset T$ is Borel in $S$ then $B$ is Borel in $T$ and

$$
\begin{aligned}
& \mu * \nu(B)=\iint \xi_{B}(x y) d \mu(x) d \nu(y) \\
= & \int_{T} \int_{T} \xi_{B}(x y) d \mu(x) d \nu(y)+\iint_{T} \int_{T^{\prime}} \xi_{B}(x y) d \mu(x) d \nu(y) \\
= & \mu_{T} * \nu_{T}(B)+\iint_{T} \int_{T^{\prime}} \xi_{B}(x y) d \mu(x) d \nu(y),
\end{aligned}
$$

since $T$ is a subsemigroup and $\nu\left(T^{\prime}\right)=0$.

Hence if $\left\{B_{1}, B_{2}, \cdots, B_{n}\right\}$ is a partition of $T$ into Borel sets in $S$, we have

$$
\left|(\mu * \nu-\nu)\left(B_{k}\right)\right| \leqq\left|\left(\mu_{T} * \nu_{T}-\nu_{T}\right)\left(B_{k}\right)\right|+\int_{T} \int_{T^{\prime}} \xi_{B_{k}}(x y) d \mu(x) d \nu(y)
$$

and

$$
\begin{aligned}
& |\mu * \nu-\nu|(T) \\
& =\sup \left\{\sum_{k=1}^{n}\left|(\mu * \nu-\nu)\left(B_{k}\right)\right|:\left\{B_{1}, B_{2}, \cdots, B_{n}\right\}\right. \text { a Borel } \\
& \text { partition of } T \text { in } S\} \\
& \leqq\left|\boldsymbol{\mu}_{T^{*}} \nu_{T}-\nu_{T}\right|(T)+\int_{T} \int_{T^{\prime}} \xi_{T}(x y) d \mu(x) d \nu(y) \\
& \leqq\left|\mu_{T} * \nu_{T}-\nu_{T}\right|(T)+\varepsilon \text {. }
\end{aligned}
$$

The last part of the lemma is now trivial.

\section{LEMMA 3.3.}

(1) Let $\nu_{\alpha}$ be a net in $M_{0}(T)$ such that $\left\|\nu * \nu_{\alpha}-\nu_{\alpha}\right\| \rightarrow 0$ for each $\nu \in M_{0}(T)$. If $0<\varepsilon<1$ and $\tau$ is a measure in $M^{+}(T)$ such that $1-\varepsilon<\tau(T)=\|\tau\| \leqq 1$, then there is some $\alpha_{0}$ (depending on $\tau$ and $\varepsilon$ ) such that

$$
\left\|\tau * \nu_{\alpha}-\nu_{\alpha}\right\| \leqq 2 \varepsilon \text { if } \alpha \geqq \alpha_{0} .
$$


(2) Let $\nu_{\alpha}$ be a net in $M_{0}(T)$ such that for each $F \subset T$ compact, $\left\|\nu * \nu_{\alpha}-\nu_{\alpha}\right\| \rightarrow 0$ uniformly for all $\nu \in M_{0}(T)$ with $\nu(F)=1$. Let $0<\varepsilon<1$ and $F$ a compact subset of $T$ be given. Then there is some $\alpha_{0}$ (depending on $\varepsilon$ and $F$ ) such that for any $\tau \in M^{+}(T)$ with $\tau(T \backslash F)=0$ and $1-\varepsilon<\tau(T)=\|\tau\| \leqq 1$, we have

$$
\left\|\tau * \nu_{\alpha}-\nu_{\alpha}\right\| \leqq 2 \varepsilon \text { for } \alpha \geqq \alpha_{0} \text {. }
$$

Proof.

(1) Let $c=\|\tau\| \neq 0$ and write $\tau=c \nu$ with $\nu \in M_{0}(T)$. Then $0 \leqq 1-c<\varepsilon$ and

$$
\begin{aligned}
\left\|\tau * \nu_{\alpha}-\nu_{\alpha}\right\| & =\left\|c\left(\nu * \nu_{\alpha}-\nu_{\alpha}\right)+c \nu_{\alpha}-\nu_{\alpha}\right\| \\
& \leqq\left\|\nu * \nu_{\alpha}-\nu_{\alpha}\right\|+|c-1| \\
& \leqq 2 \varepsilon \text { if } \alpha \geqq \alpha_{0} .
\end{aligned}
$$

(2) Let $0<\varepsilon<1$ and $F \subset T$ compact be given. There is some $\alpha_{0}$ (depending on $\varepsilon$ and $F$ ) such that

$$
\left\|\nu * \nu_{\alpha}-\nu_{\alpha}\right\|<\varepsilon \text { if } \alpha \geqq \alpha_{0} \text { and } \nu \in M_{0}(T)
$$

such that $\nu(T \backslash F)=0$. Let $\tau \in M^{+}(T)$ with $\tau(T \backslash F)=0$ and $1-\varepsilon<$ $\tau(T)=\|\tau\| \leqq 1$. Write $\tau=c \nu$ where $c=\|\tau\| \neq 0$ and $\nu \in M_{0}(T)$. Then as before $0 \leqq 1-c<\varepsilon$ and $\nu(T \backslash F)=0$, and

$$
\begin{aligned}
\left\|\tau * \nu_{\alpha}-\nu_{\alpha}\right\| & \leqq\left\|\nu * \nu_{\alpha}-\nu_{\alpha}\right\|+|c-1| \\
& \leqq 2 \varepsilon \text { if } \alpha \geqq \alpha_{0} .
\end{aligned}
$$

THEOREM 3.4. Let $S$ be a locally compact semigroup and $T$ a locally compact Borel subsemigroup of $S$ satisfying condition (*) of $\S 2$, then $S$ is topological left amenable iff $T$ is topological left amenable.

Proof. Assume that $S$ is topological left amenable (i.e., $M(S)^{*}$ has a topological left invariant mean). Since $T$ satisfies $\left(^{*}\right)$, by Theorem 3.1, there is a topological left invariant mean $M$ on $M(S)^{*}$ such that $M\left(\chi_{T}\right)=1$. Therefore $M(T)^{*}$ also has a topological left invariant mean by a topological analogue (separately continuous version) of Day's well-known criterion for amenability of (discrete) subsemigroups (Day [1] and Wong [14, Theorem 4.1]).

Conversely, suppose $M(T)^{*}$ has a topological left invariant mean. Then there is a net $\nu_{\alpha}$ in $M_{0}(T)$ such that $\left\|\tau * \nu_{\alpha}-\nu_{\alpha}\right\| \rightarrow 0$ for each $\tau \in M_{0}(T)$. Let $\mu_{\alpha}$ be the unique measure in $M_{0}(T)$ with $\mu_{\alpha}\left(T^{\prime}\right)=0$ and $\mu_{\alpha \mid T}=\nu_{\alpha}$. Suppose now $\nu \in M_{0}(S)$ has compact support. We claim that $\left\|\nu * \mu_{\alpha}-\mu_{\alpha}\right\| \rightarrow 0$. By $\left(^{*}\right)$, given $0<\varepsilon<1$, there is some $\mu \in M_{0}(S)$ with compact support such that 


$$
\mu(T)>1-\varepsilon \text { and } \nu * \mu(T)>1-\varepsilon
$$

(i.e., $\mu\left(T^{\prime}\right)<\varepsilon$ and $\left.\nu * \mu\left(T^{\prime}\right)<\varepsilon\right)$.

Now apply Lemma 3.3 (1) to the measures $\tau=\mu_{T}$ and $(\nu * \mu)_{T}$, there is some $\alpha_{0}$ such that if $\alpha \geqq \alpha_{0}$

$$
\left\|\mu_{T} * \nu_{\alpha}-\nu_{\alpha}\right\|<2 \varepsilon
$$

and

$$
\left\|(\nu * \mu)_{T} * \nu_{\alpha}-\nu_{\alpha}\right\|<2 \varepsilon .
$$

By Lemma 3.2, if $\alpha \geqq \alpha_{0}$

$$
\begin{aligned}
\left\|\mu * \mu_{\alpha}-\mu_{\alpha}\right\| & \leqq\left\|\mu_{T} * \mu_{\alpha \mid T}-\mu_{\alpha \mid T}\right\|+2 \varepsilon \\
& \leqq\left\|\mu_{T} * \nu_{\alpha}-\nu_{\alpha}\right\|+2 \varepsilon \leqq 4 \varepsilon
\end{aligned}
$$

and similarly

$$
\begin{aligned}
\left\|(\nu * \mu) * \mu_{\alpha}-\mu_{\alpha}\right\| & \leqq\left\|(\nu * \mu)_{T} * \mu_{\alpha \mid T}-\mu_{\alpha \mid T}\right\| \\
& \leqq\left\|(\nu * \mu)_{T} * \nu_{\alpha}-\nu_{\alpha}\right\|+2 \varepsilon \\
& \leqq 4 \varepsilon
\end{aligned}
$$

Consequently,

$$
\begin{aligned}
& \left\|\nu * \mu_{\alpha}-\mu_{\alpha}\right\| \\
& \quad \leqq\left\|\nu * \mu_{\alpha}-\nu *\left(\mu * \mu_{\alpha}\right)\right\|+\left\|(\nu * \mu) * \mu_{\alpha}-\mu_{\alpha}\right\| \\
& \quad \leqq\left\|\mu * \mu_{\alpha}-\mu_{\alpha}\right\|+\left\|(\nu * \mu) * \mu_{\alpha}-\mu_{\alpha}\right\| \\
& \quad \leqq 8 \varepsilon \text { if } \alpha \geqq \alpha_{0} .
\end{aligned}
$$

Therefore $S$ is topological left amenable and this completes the proof.

REMARKS. Theorem 3.4 is an extension of Wong [10, Theorem 3.2, p. 233].

4. Uniform strong topological left amenability. It is quite natural to ask whether Mitchell's second result has also an analogue for uniform strong topological left amenability. To answer this in affirmative, we need the following concept of left lumpiness first introduced by Day [4] for a Borel subset $T$ in $S$ (not necessarily a subsemigroup):

For each $K \subset S$ compact, there is some $s \in S$ such that $K s \subset T$.

Like Mitchell's concept of left thickness, there is no loss of generality here in assuming that $s \in T$. Thus we have the following string of implications

$$
(L I) \Longrightarrow(L L) \Longrightarrow(T L S) \Longrightarrow(T L T) \Longrightarrow(*) \Longrightarrow(T L L) \Longrightarrow(L T)
$$


with $(L I)$ which stands for left ideal being the strongest and Mitchell's $(L T)$ the weakest of all these conditions.

THEOREM 4.1. Let $T$ be a locally compact Borel subsemigroup of a locally compact semigroup S. Consider the following statements:

(a) $S$ is uniform strong topological left amenable

(b) $T$ is uniform strong topological left amenable.

If $T$ satisfies (*), then (a) implies (b). If $T$ is left lumpy, then (a) and (b) are equivalent.

Proof. Suppose $T$ satisfies $\left(^{*}\right)$ and $S$ is uniform strong topological left amenable. Then by Theorem 3.1, there is a topological left invariant mean $M$ such that $M\left(\chi_{T}\right)=1$. By [11, Lemma 3.1, p. 296, (separately continuous version, same proof)], we can assume that there is a net $\mu_{\alpha} \in M_{0}(S)$ such that for each compact set $K \subset S$, $\left\|\mu_{*} \mu_{\alpha}-\mu_{\alpha}\right\| \rightarrow 0$ uniformly for $\mu \in M_{0}(S)$ with $\mu(K)=1$ and that $\mu_{\alpha} \rightarrow M$ weak in $^{*} M(S)^{* *}$. Define $\tau_{\alpha}$ and $\nu_{\alpha}$ as in the proof of Lemma 3.1 above and let $\theta_{\alpha} \in M(T)$ be defined by

$$
\int g d \theta_{\alpha}=\int g^{\prime} d \nu_{\alpha}, \quad g \in C_{0}(T)
$$

where $g^{\prime}(s)=g(s)$ if $s \in T$ and $g^{\prime}(s)=0$ if $s \notin T$. Then $\theta_{\alpha}=\nu_{\alpha \mid T} \epsilon$ $M_{0}(T)$. (See Wong [9] and [14, Lemma 3.1] (separately continuous versions).) Now let $F \subset T$ be compact and $\nu \in M_{0}(T)$ with $\nu(F)=1$. Then there is a unique $\mu \in M_{0}(S)$ with $\mu\left(T^{\prime}\right)=0$ and $\left.\mu\right|_{T}=\nu$. Clearly $\mu(F)=1$. Since $\mu\left(T^{\prime}\right)=0, \nu_{\alpha}\left(T^{\prime}\right)=0$, we have

$$
\begin{aligned}
\left\|\nu * \theta_{\alpha}-\theta_{\alpha}\right\| & =\left\|\mu_{T} * \nu_{\alpha \mid T}-\nu_{\alpha \mid T}\right\|=\left\|\left.\left(\mu * \nu_{\alpha}-\nu_{\alpha}\right)\right|_{T}\right\| \\
& =\left\|\mu * \nu_{\alpha}-\nu_{\alpha}\right\| \\
\leqq & \mu * \nu_{\alpha}-\mu * \mu_{\alpha}\|+\| \mu * \mu_{\alpha}-\mu_{\alpha}\|+\| \mu_{\alpha}-\nu_{\alpha} \| .
\end{aligned}
$$

Now $\left\|\mu_{\alpha}-\nu_{\alpha}\right\| \rightarrow 0$ and $\|\mu\|=1$, this last sum tends to zero uniformly for $\nu \in M_{0}(T)$ with $\nu(F)=1$. Hence (a) implies (b).

If $T$ satisfies $(L L)$ which is stronger than $\left({ }^{*}\right)$, then (a) certainly implies (b). Conversely, suppose $T$ is uniform strong topological left amenable. Let $\nu_{\alpha} \in M_{0}(T)$ be such that for any $F \subset T$ compact, $\left\|\nu * \nu_{\alpha}-\nu_{\alpha}\right\| \rightarrow 0$ uniformly for $\nu \in M_{0}(T)$ with $\nu(F)=1$. Let $\mu_{\alpha}$ be the unique measure in $M_{0}(S)$ such that $\mu_{\alpha \mid T}=\nu_{\alpha}$, and $\mu_{\alpha}\left(T^{\prime}\right)=0$. We claim that the net $\mu_{\alpha}$ converges strongly to topological left invariance uniformly on compacta in $S$. Let $K \subset S$ be compact. By $(L L)$, there is some $a \in T$ such that $K a \subset T$. Then $F=K a \cup$ $\{a\}$ is a compact subset of $T$. Given $\varepsilon>0$, there is some $\alpha_{0}$ depending on $(\varepsilon, F)$ such that

$$
\left\|\delta_{t} * \nu_{\alpha}-\nu_{\alpha}\right\|<\varepsilon \text { if } \alpha \geqq \alpha_{0} \text { and } t \in K a \cup\{a\} \text {. }
$$


Therefore for any $\alpha \geqq \alpha_{0}, k \in K$, we have $k a \in K a$ and

$$
\begin{aligned}
& \left\|\delta_{k} * \mu_{\alpha}-\mu_{\alpha}\right\| \\
& \quad \leqq\left\|\delta_{k} * \mu_{\alpha}-\delta_{k} * \delta_{a} * \mu_{\alpha}\right\|+\left\|\delta_{k a} * \mu_{\alpha}-\mu_{\alpha}\right\| \\
& \quad \leqq\left\|\delta_{a} * \mu_{\alpha}-\mu_{\alpha}\right\|+\left\|\delta_{k a} * \mu_{\alpha}-\mu_{\alpha}\right\| \\
& \quad=\left\|\delta_{a} * \nu_{\alpha}-\nu_{\alpha}\right\|+\left\|\delta_{k a} * \nu_{\alpha}-\nu_{\alpha}\right\|<2 \varepsilon .
\end{aligned}
$$

This implies that $S$ is uniform strong topological left amenable (See Day [4, (1sau) $\Leftrightarrow(W)$ pp. 88-89].) and the proof is complete.

If the semigroup $S$ is jointly continuous, then the result can be partially strengthened.

TheOREM 4.2. Let $T$ be a closed topological left thick subsemigroup of a jointly continuous semigroup $S$. Then $T$ is uniform strong topological left amenable iff $S$ is.

Proof. Since $T$ is closed, $T$ is necessarily locally compact Borel. Sufficiency is clear by Theorem 4.1. On the other hand, if $T$ is uniform strong topological left amenable, there is a net $\nu_{\alpha} \in M_{0}(T)$ such that for each compact $F \subset T,\left\|\nu * \nu_{\alpha}-\nu_{\alpha}\right\| \rightarrow 0$ uniformly for all $\nu \in M_{0}(T)$ with $\nu(F)=1$. Again let $\mu_{\alpha} \in M_{0}(S)$ satisfy $\mu_{\alpha}\left(T^{\prime}\right)=0$ and $\mu_{\alpha \mid T}=\nu_{\alpha}$. We claim that $\mu_{\alpha}$ converges strongly to topological left invariance uniformly on compacta. Let $K \subset S$ be compact and $0<\varepsilon<1$. By $(T L T)$, there is some $\mu_{1} \in M_{0}(S)$ with compact support $K_{1} \subset S$ such that $\mu_{1}(T)>1-\varepsilon$ and $\mu * \mu_{1}(T)>1-\varepsilon$ for all $\mu \in M_{0}(S)$ with $\mu(K)=1$. Since $T$ is closed and $S$ is jointly continuous, both $F_{1}=K_{1} \cap T$ and $F_{2}=K_{2} \cap T$ where $K_{2}=K K_{1}$ are compact subsets of $T$. So is $F=F_{1} \cup F_{2}$. By Lemma $3.3(2)$, there is some $\alpha_{0}$, depending on $(\varepsilon, F)$ such that for any $\tau \in M^{+}(T)$ with $\tau(T \backslash E)=0$ and $1-\varepsilon<\tau(T)=\|\tau\| \leqq 1$, we have

$$
\left\|\tau * \nu_{\alpha}-\nu_{\alpha}\right\|<2 \varepsilon \text { if } \alpha \geqq \alpha_{0} \text {. }
$$

Now apply this to the measures $\tau=\mu_{1 \mid T}$ and $\left(\mu * \mu_{1}\right)_{T}$ where $\mu \in M_{0}(S), \mu(K)=1$. We have

$$
1 \geqq \mu_{1 \mid T}(T)=\mu_{1}(T)>1-\varepsilon
$$

and

$$
\mu_{1 \mid T}(T \backslash F)=\mu_{1}(T \backslash F) \leqq \mu_{1}\left(T \backslash F_{1}\right)=\mu_{1}\left(T \cap K_{1}^{\prime}\right) \leqq \mu_{1}\left(K_{1}^{\prime}\right)=0,
$$

since support $\mu_{1}=K_{1}$.

Similarly,

$$
1 \geqq\left(\mu * \mu_{1}\right)_{T}(T)=\mu * \mu_{1}(T)>1-\varepsilon
$$

and 


$$
\begin{aligned}
\left(\mu * \mu_{1}\right)_{T}(T \backslash F) & =\mu * \mu_{1}(T \backslash F) \leqq \mu * \mu_{1}\left(T \backslash F_{2}\right) \\
& =\mu * \mu_{1}\left(T \cap K_{2}^{\prime}\right) \leqq \mu * \mu_{1}\left(K_{2}^{\prime}\right) \\
& =0,
\end{aligned}
$$

since

$$
\mu * \mu_{1}\left(K_{2}\right)=\iint \xi_{K K_{1}}(x y) d \mu(x) d \mu_{1}(y)=1 .
$$

Hence by Lemma $3.3(2)$,

$$
\left\|\mu_{1 \mid T} * \nu_{\alpha}-\nu_{\alpha}\right\|<2 \varepsilon
$$

and

$$
\left\|\left(\mu * \mu_{1}\right)_{T} * \nu_{\alpha}-\nu_{\alpha}\right\|<2 \varepsilon \text { for all } \alpha \geqq \alpha_{0}, \mu \in M_{0}(S) \text { with } \mu(K)=1 .
$$

Consequently, for all $\alpha \geqq \alpha_{0}, \mu \in M_{0}(S), \mu(K)=1$, we have

$$
\begin{aligned}
& \left\|\mu * \mu_{\alpha}-\mu_{\alpha}\right\| \\
& \quad=\left\|\mu * \mu_{\alpha}-\mu *\left(\mu_{1} * \mu_{\alpha}\right)\right\|+\left\|\left(\mu * \mu_{1}\right) * \mu_{\alpha}-\mu_{\alpha}\right\| \\
& \quad \leqq\left\|\mu_{1} * \mu_{\alpha}-\mu_{\alpha}\right\|+\left\|\left(\mu * \mu_{1}\right) * \mu_{\alpha}-\mu_{\alpha}\right\| \\
& \quad \leqq\left\|\mu_{1 \mid T} * \nu_{\alpha}-\nu_{\alpha}\right\|+\left\|\left(\mu * \mu_{1}\right)_{T} * \nu_{\alpha}-\nu_{\alpha}\right\|+4 \varepsilon \leqq 8 \varepsilon,
\end{aligned}
$$

by Lemma 3.2 and above. This completes the proof.

5. Pointwise strong left amenability. As mentioned in Day [4], an analogue of Theorem 1.1 is still needed for left amenable locally compact semigroups which characterizes those subsets on which some left invariant mean can be concentrated. He also remarked without proof that to obtain a left invariant mean which concentrates on a Borel subset $T$, under the assumption that $T$ is left thick, would require something like left amenability of $S$ regarded as a discrete semigroup which is not a common property of left amenable locally compact semigroups.

In this section, we shall first show that if $S$ is left amenable as a discrete semigroup, then $S$ is left amenable as a locally compact semigroup and then supply a proof of Day's remark, using an elegant application of the fixed point property for left amenable discrete semigroups. Also we shall obtain an analogue of Mitchell's second result (Theorem 1.2).

THEOREM 5.1. Let $S$ be a locally compact semigroup which is left amenable as a discrete semigroup, then $S$ is left amenable. In this case, if $T$ is a Borel subset of $S$, then the following statements are equivalent: 
(1) There is a left invariant mean $M$ on $M(S)^{*}$ such that $M\left(\chi_{T}\right)=1$.

(2) $T$ is left thick.

(3) There is a left invariant mean $m$ on $m(S)$ such that $m\left(\xi_{T}\right)=1$.

Proof. Suppose $S$ is left amenable as a discrete semigroup. Let $\varphi: B M(S) \rightarrow M(S)^{*}$ be the natural embedding of the bounded Borel measurable functions $B M(S)$ into $M(S)^{*}$ defined by $\varphi(f)(\mu)=$ $\int f d \mu, \mu \in M(S)$. It is known that $\varphi$ is an order preserving isometric isomorphism (into) which commutes with left translations and $\varphi(1)=$ 1. Let $m$ be a left invariant mean on $m(S)$ and $n$ its restriction to $B M(S)$. Then $n$ is left invariant on $B M(S)$. Let $K$ be the set of means $N$ on $M(S)^{*}$ which extends $n$. (In other words $\varphi^{*}(N)=$ n.) By Hahn-Banach Extension Theorem, $K \neq \phi$. (A mean $M$ on $M(S)^{*}$ can be defined equivalently as $M(1)=\|M\|=1$.) $K$ is a compact convex subset of the separated locally convex space $M(S)^{* *}$ with the weak* topology. Moreover, if $a \in S$ and $N \in K$, then $l_{a}^{*} N \in K$ where $l_{a} ; M(S)^{*} \rightarrow M(S)^{*}$ is the left translation operator in $M(S)^{*}$ defined by $l_{a} F=\delta_{a} \odot F$. Therefore the $\operatorname{map}(s, N) \rightarrow l_{s}^{*} N$ is an action of $S$ as continuous affine maps in $K$. By Day's Fixed Point Theorem (Day [2, Theorem 1] or Mitchell [7, Theorem 5]), this action has a fixed point $N$ which is a left invariant mean on $M(S)^{*}$ (extending $n$ ). By Day [4, Theorem, p. 91], (1) implies (2) which is equivalent to (3) by Mitchell [7, Theorem 7, p. 257]. It remains to show that (3) implies (1). This however follows from the above arguments since we can assume in the definition of $K$, the mean $n$ to satisfy $n\left(\xi_{T}\right)=1$, then any fixed point $N$ has the property that $N\left(\chi_{T}\right)=1$ because $\varphi\left(\xi_{T}\right)=\chi_{T}$. This completes the proof.

REMARKS. Theorem 5.1 is an analogue of a result in Wong [11, Theorem 5.2, p. 301] for locally compact groups.

THEOREM 5.2. Let $T$ be a locally compact Borel subsemigroup of a locally compact semigroup $S$. If $T$ satisfies ( $T L L$ ), then $T$ is left amenable iff $S$ is.

Proof. Suppose $S$ is left amenable and $T$ satisfies (TLL). Then there is a left invariant mean $M$ on $M(S)^{*}$ such that $M\left(\chi_{T}\right)=$ 1 by Day [4, Theorem, p. 91]. Hence $M(T)^{*}$ also has a left invariant mean (Wong [14, Theorem 4.2, separately continuous version]). 
Conversely, suppose $T$ is left amenable, and $\nu_{\alpha}$ is a net in $M_{0}(T)$ such that $\left\|\delta_{t} * \nu_{\alpha}-\nu_{\alpha}\right\| \rightarrow 0$ for each $t \in T$. Let $\mu_{\alpha} \in M_{0}(S)$ be such that $\mu_{\alpha}\left(T^{\prime}\right)=0$ and $\mu_{\alpha \mid T}=\nu_{\alpha}$. Since $T$ satisfies $(T L L), T$ is left thick. For $s \in S$, there is some $t \in T$ 'such that $s t \in T$. Consequently

$$
\begin{aligned}
& \left\|\delta_{s} * \mu_{\alpha}-\mu_{\alpha}\right\| \\
& \quad \leqq\left\|\delta_{s} * \delta_{t} * \mu_{\alpha}-\delta_{s} * \mu_{\alpha}\right\|+\left\|\delta_{s t} * \mu_{\alpha}-\mu_{\alpha}\right\| \\
& \quad \leqq\left\|\delta_{t} * \mu_{\alpha}-\mu_{\alpha}\right\|+\left\|\delta_{s t} * \mu_{\alpha}-\mu_{\alpha}\right\| \\
& \quad=\left\|\delta_{t} * \nu_{\alpha}-\nu_{\alpha}\right\|+\left\|\delta_{s t} * \nu_{\alpha}-\nu_{\alpha}\right\| \rightarrow 0 .
\end{aligned}
$$

Hence $S$ is left amenable.

6. Some examples.

(1) Let $S=R$ be the real numbers under addition and usual topology. Then $S$ is a locally compact abelian group. $S$ is amenable in every sense we have considered. Let $T$ be either $[0, \infty)$ or $(0, \infty)$, then $T$ is a locally compact Borel subsemigroup of $S$ which is clearly left lumpy in $S$. Therefore by Theorem 4.1, $T$ is uniform strong topological left amenable.

(2) Let $S$ be a compact semigroup with identity. Suppose $C B(S)$, the continuous bounded functions on $S$ has a left invariant mean. By DeLeeuw and Glicksberg [5, Lemma 2.8, p. 70], $S$ has a unique minimal right ideal, the kernel $K(S)$ of $S$ which is a disjoint union of minimal left ideals of $S$ that are compact topological groups. Let $T$ be any one of these. Then $T$ is left lumpy. Being a compact group, $T$ is uniform strong topological left amenable. By Theorem 4.1, so is $S$. On the other hand if $M(S)^{*}$ has a left invariant mean, so does $C B(S)$ by restriction. It follows that for compact semigroups with identity, uniform strong topological left amenability, the existence of topological left invariant mean or left invariant mean on $M(S)^{*}$ or $C B(S)$ are all equivalent. [Note that the restriction of the natural embedding $\varphi: B M(S) \rightarrow M(S)^{*}$ to $C B(S)$ commutes (besides with left translations) also with left convolutions: $\varphi(\mu \odot f)=\mu \odot \varphi(f)$ if $f \in C B(S)$ and $\mu \in M(S)$, while if $f \in B M(S)$ and $\mu \in M(S), \mu \odot f$ need not be in $B M(S)$ but is in $G L(S)$, the generalized functions on $S$ (See Wong [13] for details).] In fact, we can show that any left invariant mean $m$ on $C B(S)$ is always topological left invariant. For with notations as above, let $\nu$ be the normalised left Haar measure in $T$. Again by [5, Lemma 2.8, p. 70], $m(f)=\int f_{\mid T} d \nu, f \in C B(S)$. Let $\mu \in M_{0}(S)$ be such that $\mu\left(T^{\prime}\right)=$ 0 and $\mu_{i_{T}}=\nu$. By Wong [9, Lemma 3.3, p. 129], $\delta_{a} * \mu=\mu$ for all $a \in T$. Since $T$ is a left ideal in $S, \delta_{s} * \mu=\mu$ for all $s \in S$. It follows 
that $\tau * \mu=\mu$ for all $\tau \in M_{0}(S)$. Moreover $m(\tau \odot f)=\int(\tau \odot f)_{T} d \nu=$ $\int \tau \odot f d \mu=\int f d \tau * \mu=\int f d \mu=\int f_{\mid T} d \nu=m(f)$ for any $\tau \in M_{0}(S)$ and $m$ is topological left invariant. [Recall that $\tau \odot f(x)=\int f(y x) d \tau(y)$ for $f \in C B(S), \tau \in M(S)$.]

Addendum. After the submission of the present paper, we have been informed by M.M. Day that in general the measure $\mu$ in condition $(*)$ can be chosen such that $\mu(T)=1$ and that as a consequence, topological left lumpiness is equivalent to condition $(*)$. This latter result was also communicated to us independently by H. Junghenn.

M. M. Day also claims that if a Borel subset $T$ is topological left substantial, then $T^{-}$is left lumpy and as a consequence, these two concepts coincide for closed sets.

\section{REFERENCES}

1. M. M. Day, Amenable semigroups, Illinois J. Math., 1 (1957), 509-544.

2. - Fixed point theorems for compact convex sets, Illinois J. Math., 5 (1961), 585-590; also Correction, same journal, 8 (1964), 713.

3. - Semigroups and amenability, Proceedings, Symposium on Semigroups, Wayne State University, edited by K. W. Folley, Academic Press, New York and London, (1969), 5-54.

4. - Lumpy subsets in left-amenable locally compact semigroups, Pacific J. Math., 62 (1976), 87-92.

5. K. DeLeeuw and I. Glicksberg, Application of almost periodic compactifications, Acta Math., 105 (1961), 63-67.

6. E. Hewitt and K. A. Ross, Abstract Harmonic Analysis I, Springer-Verlag, Berlin, 1963.

7. T. Mitchell, Constant functions and left invariant means on semigroups, Trans. Amer. Math. Soc., 119 (1965), 244-261.

8. James C. S. Wong, An ergodic property of locally compact amenable semigroups, Pacific J. Math., 48 (1973), 615-619.

9. — Absolutely continuous measures on locally compact semigroups, Canad. Math. Bull., 18 (1975), 127-132.

10. - Amenability and substantial semigroups, Canad. Math. Bull., 19 (1976), 231-234.

11. - A characterization of topological left thick subsets in locally compact left amenable semigroups, Pacific J. Math., 62 (1976), 295-303.

12. - Convolution and separate continuity, Pacific J. Math., 75 (1978), 601-611.

13. - Abstract harmonic analysis of generalized functions on locally compact semigroups with applications to invariant means, J. Austral. Math. Soc., 23 (Series A) (1978), 84-94.

14. - A characterization of locally compact amenable subsemigroups, to appear in Canad. Math. Bull.

Received May 3, 1978 and in revised form March 8, 1979. Research supported by the National Research Council of Canada Grant No. A8227.

UNIVERSITY OF CALGARY

Calgary, Canada T2N 1N4 



\title{
PACIFIC JOURNAL OF MATHEMATICS
}

\section{EDITORS}

DONALD BABBITT (Managing Editor)

University of California

Los Angeles, CA 90024

\section{HUGO RossI}

University of Utah

Salt Lake City, UT 84112

C. C. MOORE and ANDREW OGG

University of California

Berkeley, CA 94720

\section{J. DUGUNDJI}

Department of Mathematics University of Southern California Los Angeles, CA 90007

R. FINN and J. Milgram

Stanford University Stanford, CA 94305

\section{ASSOCIATE EDITORS}
E. F. BECKENBACH
B. H. NeUmanN
F. WOLF
K. YosHIDA

\section{SUPPORTING INSTITUTIONS}

\author{
UNIVERSITY OF BRITISH COLUMBIA \\ CALIFORNIA INSTITUTE OF TECHNOLOGY \\ UNIVERSITY OF CALIFORNIA \\ MONTANA STATE UNIVERSITY \\ UNIVERSITY OF NEVADA, RENO \\ NEW MEXICO STATE UNIVERSITY \\ OREGON STATE UNIVERSITY \\ UNIVERSITY OF OREGON
}

\author{
UNIVERSITY OF SOUTHERN CALIFORNIA \\ STANFORD UNIVERSITY \\ UNIVERSITY OF HAWAII \\ UNIVERSITY OF TOKYO \\ UNIVERSITY OF UTAH \\ WASHINGTON STATE UNIVERSITY \\ UNIVERSITY OF WASHINGTON
}

The Supporting Institutions listed above contribute to the cost of publication of this Journal, but they are not owners or publishers and have no responsibility for its content or policies.

Mathematical papers intended for publication in the Pacific Journal of Mathematics should be in typed form or offset-reproduced, (not dittoed), double spaced with large margins. Please do not use built up fractions in the text of the manuscript. However, you may use them in the displayed equations. Underline Greek letters in red, German in green, and script in blue. The first paragraph or two must be capable of being used separately as a synopsis of the entire paper. Please propose a heading for the odd numbered pages of less than 35 characters. Manuscripts, in triplicate, may be sent to any one of the editors. Please classify according to the scheme of Math. Reviews, Index to Vol. 39. Supply name and address of author to whom proofs should be sent. All other communications should be addressed to the managing editor, or Elaine Barth, University of California, Los Angeles, California, 90024.

50 reprints to each author are provided free for each article, only if page charges have been substantially paid. Additional copies may be obtained at cost in multiples of 50 .

The Pacific Journal of Mathematics is issued monthly as of January 1966. Regular subscription rate: $\$ 84.00$ a year (6 Vols., 12 issues). Special rate: $\$ 42.00$ a year to individual members of supporting institutions.

Subscriptions, orders for numbers issued in the last three calendar years, and changes of address should be sent to Pacific Journal of Mathematics, P.O. Box 969, Carmel Valley, CA 93924, U.S.A. Older back numbers obtainable from Kraus Periodicals Co., Route 100, Millwood, NY 10546.

PUBLISHED BY PACIFIC JOURNAL OF MATHEMATICS, A NON-PROFIT CORPORATION

Printed at Kokusai Bunken Insatsusha (International Academic Printing Co., Ltd.). 8-8, 3-chome, Takadanobaba, Shinjuku-ku, Tokyo 160, Japan.

Copyright (C) 1979 by Pacific Journal of Mathematics Manufactured and first issued in Japan 


\section{Pacific Journal of Mathematics}

\section{Vol. 83, No. $2 \quad$ April, 1979}

Patrick Robert Ahern, On a theorem of Hayman concerning the derivative of a

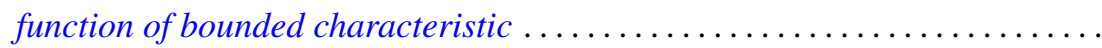

Walter Allegretto, Finiteness of lower spectra of a class of higher order elliptic

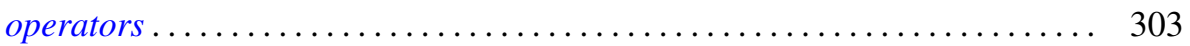

Leonard Asimow, Superharmonic interpolation in subspaces of $C_{c}(X) \ldots \ldots 11$

Steven F. Bellenot, An anti-open mapping theorem for Fréchet spaces . . . . . . . 325

B. J. Day, Locale geometry. . . . . . . . . . . . . . . . . . . . . . . . . . 333

John Erik Fornaess and Steven Krantz, Continuously varying peaking

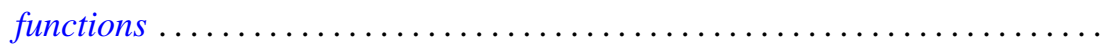

Joseph Leonide Gerver, Long walks in the plane with few collinear points ......

Joseph Leonide Gerver and Lawrence Thom Ramsey, On certain sequences of

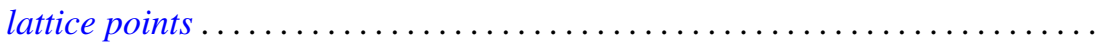

John R. Graef, Yuichi Kitamura, Takaŝi Kusano, Hiroshi Onose and Paul Winton

Spikes, On the nonoscillation of perturbed functional-differential

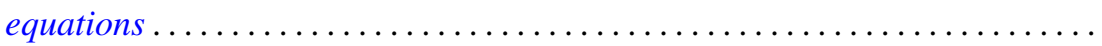

James A. Huckaba and James M. Keller, Annihilation of ideals in commutative



Anzelm Iwanik, Norm attaining operators on Lebesgue spaces . . . . . . . . . . .

Surjit Singh Khurana, Pointwise compactness and measurability . . . .......... 387

Charles Philip Lanski, Commutation with skew elements in rings with

involution.

Hugh Bardeen Maynard, A Radon-Nikodým theorem for finitely additive bounded

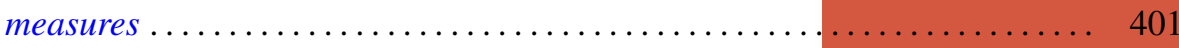

Kevin Mor McCrimmon, Peirce ideals in Jordan triple systems ..

Sam Bernard Nadler, Jr., Joseph E. Quinn and N. Stavrakas, Hyperspaces of compact convex sets.

Ken Nakamula, An explicit formula for the fundamental units of a real pure

sextic number field and its Galois closure ............

Vassili Nestoridis, Inner functions invariant connected components . .

Vladimir I. Oliker, On compact submanifolds with nondegenerate parallel

normal vector fields.

Lex Gerard Oversteegen, Fans and embeddings in the plane.

Shlomo Reisner, On Banach spaces having the property G.L

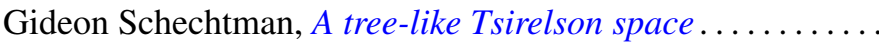

Helga Schirmer, Fix-finite homotopies . . . . . . . . . . . .

Jeffrey D. Vaaler, A geometric inequality with applications to linear forms . .

William Jennings Wickless, $T$ as an $\mathscr{G}$ submodule of $G$.....

Kenneth S. Williams, The class number of $Q(\sqrt{-p})$ modulo 4 , for $p \equiv 3$ (

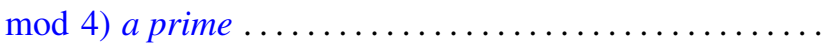

